\title{
Efeito do armazenamento na qualidade fisiológica das sementes de populações de milho cultivadas sob estresses hídrico e mineral(1)
}

\author{
Rogério Carvalho Guarçoni(2), Frederico Ozanan Machado Durães ${ }^{(3)}$, \\ Paulo César Magalhães ${ }^{(3)}$ e Roberto Ferreira da Silva(2)
}

\begin{abstract}
Resumo - O objetivo deste trabalho foi avaliar o efeito do armazenamento na qualidade fisiológica de sementes de cinco populações de milho submetidas aos estresses de Al no solo e de seca no florescimento. Cinco populações de milho (CMS06, CMS35, CMS36, CMS54 e Milho Metro) foram cultivadas em solos com dois níveis de fertilidade: o primeiro, apresentando alta saturação de bases ( $\mathrm{V}=67 \%)$, e o segundo, alta saturação de $\mathrm{Al}(\mathrm{m}=54 \%)$. As populações de milho foram também submetidas à irrigação plena e à supressão de irrigação durante o florescimento. As sementes obtidas dessas populações foram armazenadas no laboratório de sementes sob condições ambientais. A qualidade físiológica das sementes foi determinada no início e aos 30,90 e 210 dias de armazenamento. As sementes da população CMS36 apresentaram maior vigor quando produzidas em solos com alta saturação de alumínio. A população CMS54 foi a mais estável com relação à qualidade fisiológica das sementes produzidas em diferentes ambientes. As sementes das populações CMS35, CMS36, CMS54 e Milho Metro foram mais estáveis com relação à qualidade fisiológica, durante o período de armazenamento, do que as da CMS06
\end{abstract}

Termos para indexação: alumínio, solo, seca, germinação, longevidade das sementes.

Influence of storage on physiological quality of the seeds of maize populations under moisture and mineral stresses

\begin{abstract}
The objective of this work was to evaluate the storage effect on seed physiological quality of five tropical maize (Zea mays L.) populations, under stress conditions, including acid soil and drought. Five maize populations (CMS06, CMS35, CMS36, CMS54 and Milho Metro) were cultivated in soils with two types of fertility ( $67 \%$ of base saturation and $54 \%$ of Al saturation), with and without irrigation during flowering. After harvest, the seeds were stored in the laboratory under environmental conditions. The physiological quality of seeds, germination and vigor, were determined in the start of the storage, 30, 90 and 210 days after starting storage. The seeds of the population CMS36 presented higher vigor when produced under high aluminum saturation. The population CMS54 presented highest stability related to physiological quality. The seeds of the populations CMS35, CMS36, CMS54 and Milho Metro presented higher stability related to physiological quality than the CMS06, during storage.
\end{abstract}

Index terms: aluminum, soil, drought, germination, seed longevity.

(1) Aceito para publicação em 24 de novembro de 2000 . Extraído da Tese de Doutorado apresentada pelo primeiro autor à Universidade Estadual do Norte Fluminense (UENF), Campos dos Goytacazes, RJ.

(2)UENF, Av. Alberto Lamego, 2000, CEP 28015-620 Campos dos Goytacazes, RJ. E-mail: rguar@bol.br, roberto@uenf.br

${ }^{(3)}$ Embrapa-Centro Nacional de Pesquisa de Milho e Sorgo, Caixa Postal 151, CEP 35700-970 Sete Lagoas, MG. E-mail: fduraes@cnpms.embrapa.br, pcesar@cnpms.embrapa.br

\section{Introdução}

O Brasil possui 1,8 milhão de $\mathrm{km}^{2}$ de Cerrados (Magnavaca \& Bahia Filho, 1995), e a importância desses solos na produção de grãos é significativa. Nesses solos, os rendimentos são limitados pela baixa disponibilidade de $\mathrm{P}, \mathrm{Ca}$ e $\mathrm{Mg}$ e pela toxidez de Al e Mn (Baligar et al., 1997). Além disso, muitas 
regiões com esse tipo de solo freqüentemente sofrem um veranico no mês de janeiro. Assim, de acordo com Spaner et al. (1995), a produção de milho é limitada pelas condições de estresses, que são comuns em regiões tropicais, incluindo solos ácidos e seca.

Os estresses durante o desenvolvimento da cultura também podem prejudicar a viabilidade das sementes no armazenamento. $\mathrm{O}$ armazenamento prolongado das sementes de milho pode ser prejudicial a sua qualidade, pois inicia um processo degenerativo que leva à perda da sua viabilidade (Lin, 1988). O processo degenerativo das sementes é acentuado quando ocorrem condições ambientais adversas no período de produção (Dornbos et al., 1989).

El-Forgany \& Makus (1979) avaliaram o efeito da deficiência hídrica sobre o rendimento e a qualidade fisiológica das sementes de milho-doce. A linhagem Luther Hill (Zea mays L.) foi submetida a condições de solo com $\psi_{\mathrm{m}}=-0,3 \mathrm{MPae} \psi_{\mathrm{m}}=0,5 \mathrm{MPa}$, durante 3 e 6 semanas após florescimento. Observouse que esses estresses após o florescimento reduziram significativamente o rendimento e o índice de vigor das sementes, em todos os tratamentos.

Lin (1988), estudando o efeito do período de armazenamento na qualidade fisiológica da semente de milho (Zea mays L.) sob condições de estocagem de $25^{\circ} \mathrm{Ce} 79 \%$ de umidade relativa do ar por 92 dias, observou que a germinação e o vigor das sementes decresceram com o aumento do período de armazenamento. As sementes perderam completamente a viabilidade após 92 dias de armazenamento. $\mathrm{O}$ vigor das sementes decresceu mais rapidamente do que a viabilidade durante $\mathrm{o}$ armazenamento. $\mathrm{O}$ mesmo autor acrescentou que as sementes aumentaram o seu teor de água durante o período de armazenamento, em razão da absorção da água da atmosfera, e que esta absorção poderia indicar as mudanças deletérias da membrana plasmática, contribuindo com a perda do vigor e da viabilidade da semente de milho armazenada.

Segundo Puzzi (1989), o processo respiratório é acompanhado pelo consumo das substâncias de reserva, e os principais fatores que alteram a intensidade do processo respiratório são a temperatura, grau de umidade das sementes e os fungos na massa de sementes. O processo respiratório nas sementes armazenadas é acelerado pela própria reação, a qual aumenta o grau de umidade do produto e a temperatura. Silva (1995) acrescentou que danos causados às sementes no processo de deterioração são: perda da germinação, descoloração, aumento do teor de ácidos graxos das sementes, e degradação da qualidade nutritiva.

Este trabalho teve como objetivo avaliar, durante o armazenamento, a qualidade fisiológica das sementes de cinco populações de milho, cultivadas em condições de estresse hídrico no florescimento, e alta saturação em Al no solo.

\section{Material e Métodos}

O presente trabalho foi conduzido na Embrapa-Centro Nacional de Pesquisa de Milho e Sorgo, em Sete Lagoas, MG. Cinco populações de milho CMS06, CMS35, CMS36, CMS54 e Milho Metro foram cultivadas em Latossolo Vermelho-Escuro, fase cerrado, textura média, com dois níveis de fertilidade, ou seja: o primeiro, com alta saturação de bases (V=67\%), e o segundo, com alta saturação em $\mathrm{Al}$ no solo $(\mathrm{m}=54 \%)$. Em cada nível de fertilidade, foi conduzido com irrigação plena e com estresse hídrico durante o florescimento (15 dias, antes e depois do florescimento). Quatro experimentos foram montados distantes $50 \mathrm{~m}$ um do outro. No primeiro experimento, as plantas das populações foram submetidas a condições de alta saturação de bases no solo e de irrigação plena. No segundo, a saturação de bases foi a mesma do primeiro experimento, mas com supressão de irrigação no período de florescimento. No terceiro experimento, as plantas foram submetidas a condições de solo com alta saturação em Al e de irrigação plena. No quarto experimento, a saturação em $\mathrm{Al}$ foi a mesma do terceiro experimento, mas com supressão de irrigação durante o florescimento.

A unidade experimental foi constituída de seis linhas de seis metros de comprimento, a espaços de $0,9 \mathrm{~m}$ entre linhas e 0,2 m entre plantas, contendo um total de 180 plantas/parcela, com estande de 55.000 plantas/ha

A correção do solo e a adubação foram realizadas de acordo com a Comissão de Fertilizantes do Solo do Estado de Minas Gerais (1989), a partir da análise do solo. Observou-se, no nível de fertilidade de alta saturação de bases: $\mathrm{pH} 6,2 ; \mathrm{H}+\mathrm{Al}, \quad 2,65 \mathrm{meq} / 100 \mathrm{~cm}^{3} ; \mathrm{Al}$, $0,0 \mathrm{meq} / 100 \mathrm{~cm}^{3} ; \quad \mathrm{Ca}, \quad 4,26 \mathrm{meq} / 100 \mathrm{~cm}^{3} ; \quad \mathrm{Mg}$, 0,72 meq $/ 100 \mathrm{~cm}^{3} ; \mathrm{K}, 113$ ppm; P, 7 ppm; M.O., 2,70\%; $\mathrm{m}, 0 \% ; \mathrm{V}, 67 \%$. No nível de alta saturação em $\mathrm{Al}$, foram observados: $\mathrm{pH} 4,9 ; \mathrm{H}+\mathrm{Al}, 6,01 \mathrm{meq} / 100 \mathrm{~cm}^{3} ; \mathrm{Al}$, $1,50 \mathrm{meq} / 100 \mathrm{~cm}^{3} ; \mathrm{Ca}, 0,98 \mathrm{meq} / 100 \mathrm{~cm}^{3} ; \mathrm{Mg}$, $0,12 \mathrm{meq} / 100 \mathrm{~cm}^{3}$; K, 66 ppm; P, 16 ppm; M.O., 3,88\%; 
m, 54\%; V, 17\%. Foram feitas três adubações: uma, de semeadura, e duas, de cobertura. $\mathrm{Na}$ adubação de semeadura, foram utilizados $400 \mathrm{~kg} / \mathrm{ha}$ do formulado 8-28-16+ Zn. Foram realizadas duas adubações de cobertura de $\mathrm{N}$ aos 27 e 40 dias após plantio, utilizando-se $45 \mathrm{~kg} / \mathrm{ha}$ de N

Foram instalados, em cada experimento, oito tensiômetros com manômetros de mercúrio, para determinação da tensão da água no solo. Sempre que os tensiômetros apresentavam leituras de tensão de $55 \mathrm{~cm}$ de $\mathrm{Hg}$, o sistema de irrigação era acionado, para repor a água evapotranspirada (Bernardo, 1987), exceto nos experimentos em que a irrigação foi suspensa no período de florescimento.

Foi calculado o balanço hídrico durante os 30 dias sem irrigação dos experimentos de seca, para medir a intensidade do estresse. O balanço hídrico foi determinado em função da irrigação, da chuva (Tabela 1) e da evapotranspiração. A evapotranspiração diária foi estimada pela evaporação diária do tanque classe $\mathrm{A}$, do coeficiente de tanque $(\mathrm{kt})$, e do coeficiente de cultura $(\mathrm{kc})$ (Doorenbos \& Pruitt, 1977).

Foi realizada colheita manual das espigas com as sementes apresentando grau de umidade médio de $18 \%$. As espigas foram secadas à sombra até as sementes apresentarem $16 \%$ de umidade, para a realização da debulha mecânica. Após a debulha, as sementes foram secadas à sombra, até o início do armazenamento.

Em pós-colheita, as amostras de sementes das cinco populações foram armazenadas no laboratório de sementes da Embrapa-Centro Nacional de Pesquisa de Milho e Sorgo sob condições ambientais, mantendo-se as mesmas parcelas experimentais de produção. Para a análise estatística, foi utilizada análise conjunta de experimentos, em parcelas divididas, em delineamento em blocos casualizados, com três repetições. As parcelas foram constituídas pelas cinco populações de milho, e as subparcelas, constituídas por quatro épocas de análise da qualidade fisiológica durante o armazenamento. Para a avaliação da qualidade físiológica das sementes, foram realizados testes de germinação (Brasil, 1992) e vigor (Vieira \& Carvalho, 1994) após 0, 30, 90 e 210 dias de armazenamento. As sementes não receberam tratamento químico antes de serem armazenadas.

As amostras das sementes eram divididas e homogeneizadas por um divisor e homogeneizador de amostras Seedburo, antes da avaliação da qualidade fisiológica. O teste de germinação de todas as amostras foi realizado de acordo com as Regras para Análise de Sementes (Brasil, 1992). Para avaliar o vigor, foi realizado o teste de envelhecimento acelerado, utilizando o método utilizado por Vieira \& Carvalho (1994). Utilizaram-se caixas de plás- tico contendo uma tela metálica, sobre a superfície da qual foram distribuídas as sementes, distando $2 \mathrm{~cm}$ do fundo da caixa, que continha $40 \mathrm{~mL}$ de água deionizada, e em seguida foram mantidas em câmara tipo BOD, a $42 \pm 1^{\circ} \mathrm{C}$, durante 96 horas. Após o envelhecimento, quatro repetições de 100 sementes foram colocadas para germinar, segundo os critérios estabelecidos nas Regras para Análise de Sementes (Brasil, 1992).

Foi determinado o grau de umidade médio das sementes após 0, 30, 90 e 210 dias de armazenamento, utilizando o aparelho Geole, e foram monitoradas, mensalmente, a umidade relativa e a temperatura do ambiente.

\section{Resultados e Discussão}

As análises de variância da germinação e do vigor são apresentadas na Tabela 2 .

As sementes das populações CMS06, CMS35 e Milho Metro apresentaram um decréscimo significativo de germinação no quarto experimento (Tabelas 2 e 3). Não houve diferença estatisticamente significativa na germinação das sementes das populações CMS36 e CMS54 nos quatro experimentos. As sementes das populações CMS06, CMS35 e Milho Metro apresentaram um decréscimo no vigor no quarto experimento, e as sementes da população CMS54 não mostraram diferenças estatisticamente significativas no vigor entre os experimentos. Entretanto, sementes da população CMS36 apresentaram, em geral, maior vigor quando cultivadas em solos com alta saturação em $\mathrm{Al}(\mathrm{m}=54 \%)$ do que em solos com alta saturação de bases $(\mathrm{V}=67 \%)$. Isto, provavelmente, decorre pelo fato de este sintético apresentar tolerância ao Al (Lopes et al., 1987; Magnavaca \& Bahia Filho, 1993), induzindo-o a pro-

Tabela 1. Precipitação $(\mathrm{mm})$ durante os 30 dias sem irrigação nos experimentos de seca.

\begin{tabular}{cccccc}
\hline Dia & Precipitação & Dia & Precipitação & Dia & Precipitação \\
\hline 1 & 0,6 & 11 & 0,0 & 21 & 0,0 \\
2 & 0,0 & 12 & 0,0 & 22 & 0,0 \\
3 & 4,3 & 13 & 0,0 & 23 & 0,0 \\
4 & 0,0 & 14 & 9,1 & 24 & 0,0 \\
5 & 0,0 & 15 & 0,5 & 25 & 0,0 \\
6 & 0,0 & 16 & 0,0 & 26 & 0,0 \\
7 & 0,0 & 17 & 0,0 & 27 & 0,0 \\
8 & 0,0 & 18 & 0,0 & 28 & 0,0 \\
9 & 0,0 & 19 & 0,0 & 29 & 0,0 \\
10 & 1,9 & 20 & 0,0 & 30 & 0,0 \\
\hline
\end{tabular}

Pesq. agropec. bras., Brasília, v. 36, n. 12, p. 1479-1484, dez. 2001 
duzir com mais eficiência em condições de solos com alta saturação em alumínio.

Houve tendência de decréscimo na germinação a partir de 90 dias após o armazenamento, nos experimentos 1,2 e 3 , e a partir de 30 dias, no experimento 4 (Figura 1). Verificou-se também uma tendência de decréscimo no vigor, a partir de 90 dias após o armazenamento, e nos níveis de irrigação (Figura 2) e de fertilidade (Figura 3). Estas tendências podem ser explicadas pelo processo degenerativo que as sementes sofreram nas condições ambientais adversas, como umidade relativa e temperatura desfavoráveis, ocorridas durante o armazenamento das sementes, entre 90 e 210 dias (Tabela 4). Este resultado pode ser confirmado pelo teor de água alcançado pelas sementes após 210 dias de armazenamento. Os resultados estão de acordo com os de Lin (1988), que observou que o vigor e a germinação das semen-

Tabela 2. Análise de variância para germinação e vigor de sementes de milho. Sete Lagoas, MG, 1998.

\begin{tabular}{|c|c|c|c|c|c|}
\hline \multirow[t]{2}{*}{ Causa de variação } & \multirow[t]{2}{*}{ G.L. } & \multicolumn{2}{|c|}{ Germinação } & \multicolumn{2}{|c|}{ Vigor } \\
\hline & & Q.M. & $\mathrm{F}$ & Q.M. & $\mathrm{F}$ \\
\hline Bloco (ambientes) & 8 & $1.546,596$ & & $1.483,217$ & \\
\hline Ambientes & 3 & $3.689,478$ & $3,90^{*}$ & $2.607,771$ & 2,61 \\
\hline População & 4 & $2.907,204$ & 3,07 & $5.260,548$ & $5,26^{*}$ \\
\hline Ambiente $x$ população & 12 & 944,540 & $2,13 *$ & 998,656 & $2,59 *$ \\
\hline Resíduo (a) & 32 & 443,278 & & 385,498 & \\
\hline Total de parcelas & 59 & & & & \\
\hline $\mathrm{CV}(\%)$ & & 28,0 & & 30,6 & \\
\hline Época & 3 & $4.342,478$ & $46,51 *$ & $14.481,18$ & $176,56^{*}$ \\
\hline Ambiente x época & 9 & 258,622 & $2,77^{*}$ & 304,586 & $3,71 *$ \\
\hline População x época & 12 & 170,721 & 1,82 & 308,859 & $3,76^{*}$ \\
\hline Ambiente $\mathrm{x}$ população $\mathrm{x}$ época & 36 & 146,379 & $1,56^{*}$ & 131,027 & $1,59 *$ \\
\hline Resíduo (b) & 120 & 93,364 & & 82,014 & \\
\hline Total & 239 & & & & \\
\hline $\mathrm{CV}(\%)$ & & 12,8 & & 14,1 & \\
\hline
\end{tabular}

Tabela 3. Médias de germinação (\%) e de vigor (\%) de sementes de milho provenientes de cinco populações cultivadas em solos corrigido e não corrigido, com irrigação plena e sem irrigação plena na época do florescimento. Sete Lagoas, MG, 1998(1)

\begin{tabular}{llcccc}
\hline População & \multicolumn{2}{c}{ Solo corrigido } & & \multicolumn{2}{c}{ Solo não corrigido } \\
\cline { 2 - 3 } \cline { 5 - 6 } & Irrigado & Não irrigado & \multicolumn{2}{c}{ Irrigado } & Não irrigado \\
\hline & & \multicolumn{2}{c}{ Germinação } & \\
CMS06 & $77,50 \mathrm{Aab}$ & $77,66 \mathrm{Aa}$ & & $86,50 \mathrm{Aa}$ & $48,66 \mathrm{Bc}$ \\
CMS35 & $79,33 \mathrm{Aab}$ & $79,91 \mathrm{Aa}$ & & $72,25 \mathrm{Abc}$ & $66,41 \mathrm{Bb}$ \\
CMS36 & $84,33 \mathrm{Aa}$ & $79,16 \mathrm{Aa}$ & & $89,33 \mathrm{Aa}$ & $85,41 \mathrm{Aa}$ \\
CMS54 & $80,33 \mathrm{Aa}$ & $77,16 \mathrm{Aa}$ & & $85,50 \mathrm{Aab}$ & $79,25 \mathrm{Aa}$ \\
Milho Metro & $69,25 \mathrm{Ab}$ & $73,91 \mathrm{Aa}$ & & $74,58 \mathrm{Abc}$ & $39,50 \mathrm{Bc}$ \\
\hline & & & Vigor & & \\
CMS06 & $63,67 \mathrm{Abc}$ & $62,83 \mathrm{Aab}$ & & $72,17 \mathrm{Ab}$ & $43,67 \mathrm{Bb}$ \\
CMS35 & $55,92 \mathrm{Ac}$ & $54,42 \mathrm{Ab}$ & & $53,50 \mathrm{Ac}$ & $38,25 \mathrm{Bb}$ \\
CMS36 & $72,17 \mathrm{Bab}$ & $61,00 \mathrm{Cab}$ & & $85,08 \mathrm{Aa}$ & $80,75 \mathrm{ABa}$ \\
CMS54 & $75,58 \mathrm{Aa}$ & $71,00 \mathrm{Aa}$ & & $76,08 \mathrm{Aab}$ & $75,92 \mathrm{Aa}$ \\
Milho Metro & $64,58 \mathrm{Abc}$ & $69,08 \mathrm{Aa}$ & & $68,25 \mathrm{Ab}$ & $38,00 \mathrm{Bb}$ \\
\hline
\end{tabular}

${ }^{(1)}$ Médias seguidas de mesma letra, maiúscula na linha e minúscula na coluna, não diferem entre si, pelo teste de Tukey, a 5\% de probabilidade. 
tes decresceram com o período de armazenamento, e confirmam também Puzzi (1989), que observou que a absorção de água pelas sementes pode indicar sua deterioração. No experimento 4 (Figura 1), os aumentos da temperatura e da umidade relativa (Tabela 4) ocorridos a partir de 30 dias após o armazenamento, sob condições de um estresse mais severo durante a fase de produção das sementes, podem explicar a tendência de decréscimo da germinação dos 30 para os 90 dias de armazenamento.

Houve tendência de decréscimo no vigor das sementes das populações CMS35, CMS36, CMS54 e Milho Metro, a partir de 90 dias após o armazenamento, e a população CMS06 apresentou tendência de decréscimo no vigor desde o início do armazenamento (Figura 4). Os resultados indicaram

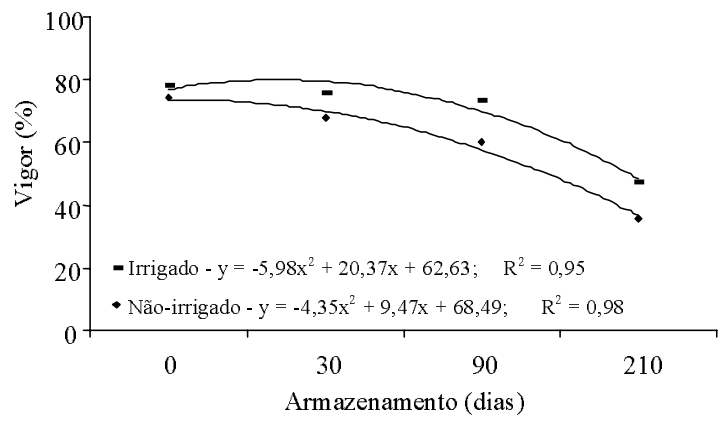

Figura 2. Equações de regressão dos resultados de vigor em diferentes períodos de armazenamento, de sementes provenientes de populações de milho cultivadas em solo irrigado e não irrigado.

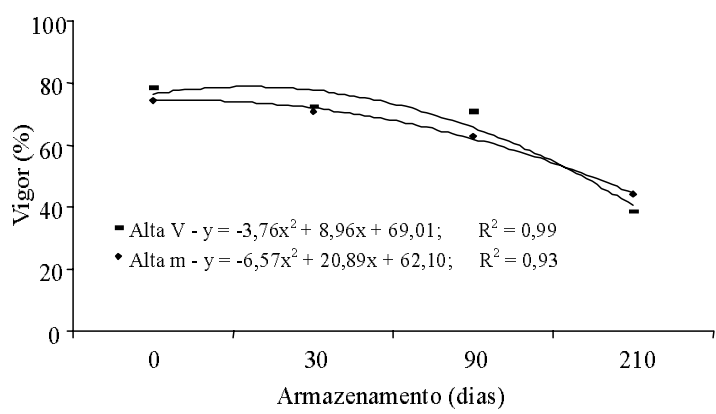

Figura 3. Equações de regressão de resultados de vigor em diferentes períodos de armazenamento, de sementes de milho, provenientes de populações cultivadas em solo com alta saturação de bases ( $\mathrm{V}=67 \%$, $\boldsymbol{\square})$ e em solo com alta saturação de alumínio $(\mathrm{m}=54 \%$,

Tabela 4. Umidade relativa (UR), temperatura de armazenamento e grau de umidade das sementes. Sete Lagoas, MG, 1998.

\begin{tabular}{cccccc}
\hline $\begin{array}{c}\text { Período de } \\
\text { armazenamento } \\
\text { (dias) }\end{array}$ & $\begin{array}{c}\text { UR }(\%) \\
\text { máxima } \\
\text { mensal }\end{array}$ & $\begin{array}{c}\text { UR }(\%) \\
\text { mínima } \\
\text { mensal }\end{array}$ & $\begin{array}{c}\text { Temperatura } \\
\text { máxima } \\
\left({ }^{\circ} \mathrm{C}\right)\end{array}$ & $\begin{array}{c}\text { Temperatura } \\
\text { mínima } \\
\left({ }^{\circ} \mathrm{C}\right)\end{array}$ & $\begin{array}{c}\text { Grau } \\
\text { de umidade } \\
(\%)\end{array}$ \\
\hline 0 & 71 & 41 & 32,0 & 8,0 & 11,3 \\
30 & 82 & 34 & 34,7 & 11,1 & 9,9 \\
60 & 92 & 37 & 35,6 & 12,8 & \\
90 & 92 & 36 & 36,6 & 18,5 & 9,0 \\
120 & 96 & 60 & 35,0 & 17,8 & \\
150 & 94 & 58 & 34,0 & 18,0 & \\
180 & 93 & 58 & 35,0 & 17,4 & 13,0 \\
210 & 85 & 59 & 33,4 & 17,8 & \\
\hline
\end{tabular}




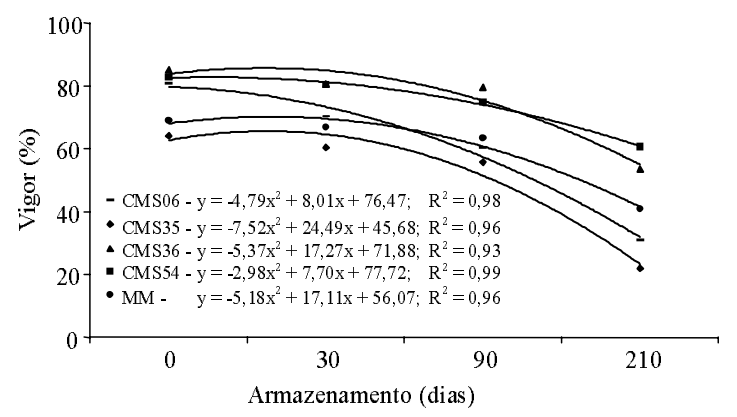

Figura 4. Equações de regressão dos resultados do teste de envelhecimento acelerado em diferentes períodos de armazenamento, em sementes de milho provenientes das populações CMS06, CMS35, CMS36, CMS54 e Milho Metro (MM).

que as sementes das populações CMS35, CMS36, CMS54 e Milho Metro são mais estáveis com relação à qualidade fisiológica, durante o armazenamento, do que as da CMSO6.

\section{Conclusões}

1. Sementes da população CMS36 apresentam maior vigor quando produzidas em solos com alta saturação em alumínio.

2. A população CMS54 é a mais estável com relação à qualidade fisiológica das sementes quando produzidas em diferentes ambientes.

3. As sementes da população CMS06 são menos estáveis quanto à qualidade fisiológica, durante o armazenamento, do que as sementes das demais populações.

\section{Referências}

BALIGAR, V. C.; PITTA, G. V. E.; GAMA, E. E. G.; SCHAFFERT, R. E.; BAHIA FILHO, A. F. C.; CLARK, $R$. B. Soil acidity effects on nutrient use efficiency in exotic maize genotypes. Plant and Soil, Dordrecht, v. 192, n. 1, p. 9-13, 1997.

BERNARDO, S. Manual de irrigação. 4. ed. Viçosa UFV, 1987. $488 \mathrm{p}$

BRASIL. Ministério da Agricultura e do Abastecimento Regras para análise de sementes. Brasília, 1992. 365 p.
COMISSÃO DE FERTILIZANTES DO SOLO DO ESTADO DE MINAS GERAIS (Lavras, MG). Recomendações para o uso de corretivos e fertilizantes em Minas Gerais: 4a aproximação. Lavras, 1989. 159 p.

DOORENBOS, J.; PRUITT, W. O. Crop water requirements. Rome: FAO, 1977. 144 p. (Irrigation and Drainage Paper, 24)

DORNBOS, D. L.; MULLEN, R. E.; SHIBLES, R. M. Drought stress effects during seed fill on soybean seed germination and vigor. Crop Science, Madison, v. 29, n. 2, p. 476-480, 1989.

EL-FORGANY, M.; MAKUS, D. J. Effect of water stress on seed yield and quality of the sweet corn inbred 'Luther Hill' Journal of the American Society for Horticultural Science, Alexandria, v. 104, n. 1, p. 102-104, 1979

LIN, S. S. Efeito do período de armazenamento na lixiviação eletrolítica dos solutos celulares e qualidade fisiológica da semente de milho (Zea mays L.) e feijão (Phaseolus vulgaris L.). Revista Brasileira de Sementes, Brasília, v. 10, n. 3, p. 59-67, 1988

LOPES, M. A.; MAGNAVACA, R.; BAHIA FILHO, A. F. C.; GAMA, E. E. G. Avaliação de populações de milho e seus cruzamentos para tolerância à toxidez de alumínio em solução nutritiva. Pesquisa Agropecuária Brasileira, Brasília, v. 22, n. 3, p. 257-263, mar. 1987.

MAGNAVACA, R.; BAHIA FILHO, A. F. C. Seleção de milho para tolerância a alumínio. In: SIMPÓSIO INTERNACIONAL SOBRE ESTRESSE AMBIENTAL: O MILHO EM PERSPECTIVA, 1992, Belo Horizonte, MG. Proceedings... Sete Lagoas: Embrapa-CNPMS/ Cimmyt-UNDP, 1995. p. 369-394.

MAGNAVACA, R.; BAHIA FILHO, A. F. C. Success in maize acid soil tolerance. In: WORKSHOP ON ADAPTATION OF PLANTS TO SOIL STRESS, 1993, Lincoln. Proceedings... Lincoln: INTSORMIL, 1993. p. $209-220$

PUZZI, D. Abastecimento e armazenagem de grãos Campinas: Instituto Campineiro de Ensino Agrícola, 1989 $603 \mathrm{p}$.

SILVA, J. S. Pré-processamento de produtos agrícolas. Juiz de Fora: Instituto Maria, 1995. 509 p.

SPANER, D.; BRATHWAITE, R. A. I.; MATHER, D. E. Comparison of open-pollinated stress-tolerant and landrace maize for production under stress conditions in Trinidad. Maydica, Bergamo, v. 40, n. 4, p. 331-337, 1995

VIEIRA, R. D.; CARVALHO, N. M. Testes de vigor em sementes. Jaboticabal: Unesp, 1994. 164 p. 